\title{
Neonatal Serum Bilirubin from Skin Reflectance
}

\author{
R. E. HANNEMANN, ${ }^{(24)}$ D. P. DEWITT, AND J. F. WIECHEL
}

School of Mechanical Engineering, Purdue University, West Lafayette, Indiana, USA

\begin{abstract}
Summary
To provide a noninvasive method for serum bilirubin determination in newborn infants, a reflectometer system was developed using a novel fiber optic arrangement permitting measurement of the light reflected from a small area $\left(3 \mathrm{~cm}^{2}\right)$ of the infant's skin. The reflectometer was operated over the spectral region $400-750 \mathrm{~nm}$ with a $30-\mathrm{sec}$ scan time. The reflectometer consisted of a monochomatic light source, fiber optic probe, detector, and data acquisition system

Spectral reflectance measurements were performed on the blanched and unblanched skin on the backs of 56 Caucasian newborn infants. Examples of the spectra and corresponding serum bilirubin values of 30 of the infants are presented, illustrating that near $460 \mathrm{~nm}$, the spectral reflectance of the skin decreases with increasing serum bilirubin concentrations. It was also observed that the individualities in epidermal hemoglobin concentration affected the skin reflectance spectra. For this reason the statistical analysis was limited to inclusion of only reflectance spectra of blanched skin.

The multispectral analysis of the data was based upon statistical regression analysis investigating the predictability of serum bilirubin level from reflectance spectra. The spectrum between 400 and $740 \mathrm{~nm}^{-}$was divided into $5-\mathrm{nm}$ intervals $(71$ discrete bands). First order linear, multiple linear, and polynomial nonlinear regressions were performed on the spectral reflectance values at each of the intervals with respect to serum bilirubin level. The coefficients of determination ( $R^{2}$ values) are summarized.

Because an $R^{2}$ of only 0.783 was observed at $450 \mathrm{~nm}$, scatter diagrams of serum bilirubin concentration versus spectral skin reflectance at a given wavelength were studied. These indicated that a logarithmic transformation of the data might yield improved results. The double natural logarithm was taken of the 71 discrete spectral reflectance values and a polynomial nonlinear regression was performed with respect to serum bilirubin concentration. The best results of this regression analysis were obtained for a combination of five wavelengths yielding an $\mathbf{R}^{2}$ value of 0.931 .
\end{abstract}

The ability to predict serum bilirubin concentration from skin reflectance using this relationship was tested using the original observations. The $95 \%$ prediction limit for this empirical relationship was shown to be $\pm 2 \mathrm{mg} / 100 \mathrm{ml}$.

\section{Speculation}

Spectral reflectance measurements on the newborn skin provide a noninvasive screening method for neonatal jaundice and may also be useful in assessing the efficacy of phototherapy and the potential for kernicterus.

The present method for detecting neonatal jaundice is visual. A positive diagnosis is verified by a blood test which measures the serum bilirubin concentration. Although the measurement of serum bilirubin concentration has been shown to be inadequate in certain instances, it currently provides the only readily available indication of the infant's potential for kernicterus. Three factors must be considered in the visual detection process: experience of the physician or nursing staff, skin pigmentation of the infant, and the nature of the environmmental lighting.

A variety of tintometers and color-matching techniques have been developed to reduce the subjective judgment of the physician $(14,18)$ but none have found widespread acceptance in the United States. This lack of acceptance is because such devices depend upon a standard environmental lighting condition and the physician's ability to make an accurate color match between the infant's skin und the color sample. For given conditions of lighting and infant pigmentation, these techniques appear to be more applicable to monitoring than detection (10).

A quantitative indication of skin color can be obtained from measurement of the color as a function of wavelength over the visible portion of the spectrum. One of the first in-depth studies of this kind was performed in 1926 by Sheard and Brown (17). Since that time, other investigators have obtained spectral reflectance data for skin using a variety of techniques and instrumentation $(2-4,6,7,9,19)$. The method of analysis used in these studies defines the color of an object by the three chromaticity coordinates (8). This is insensitive to subtle changes in spectral reflectance in narrow wavelength bands since the spectrum is essentially averaged over three wide spectral regions. Disease-oriented skin color phenomena which exhibit variations in the spectral reflectance at specific wavelength bands would not be easily detected by such a three-dimensional analysis. This explains the conclusion of Brunsting and Sheard (2) that jaundice is qualitatively detectable from the reflectance spectrum itself, but is not noticeable when using a color analysis scheme.

Recent developments in computer technology and computeraided reflectometry that facilitate a more complete analysis of reflectance spectra will be described in this paper.

\section{MATERIALS AND METHODS}

A reflectometer system, Figure 1, was developed (20) using a novel fiber optic arrangement permitting measurement of the light reflected from a small area $\left(3 \mathrm{~cm}^{2}\right)$ of the infant's skin while he remained undisturbed in his crib. The reflectometer was operated over the spectral region $400-750 \mathrm{~nm}$ with a $30-\mathrm{sec}$ scan time. The reflectometer consisted of a monochromatic light source, fiber optic probe, detector, and data acquisition system.

A tungsten light source and grating monochrometer served as a source of monochromatic light which was transmitted to the skin of the infant by one branch of the bifurcated fiber optic probe. The light reflected from the infant's skin was transmitted to a silicon photodiode detector through the second branch of the fiber optic probe. The transduced signal was amplified and recorded on one channel of an FM tape recorder. A potentiometer was coupled to the grating drive of the monochrometer and provided a wavelength reference signal which was recorded on a second channel of the FM tape recorder. The recorded analog signals were digitized and partially processed using a CDC 1700 computer facility, not shown in Figure 1. 


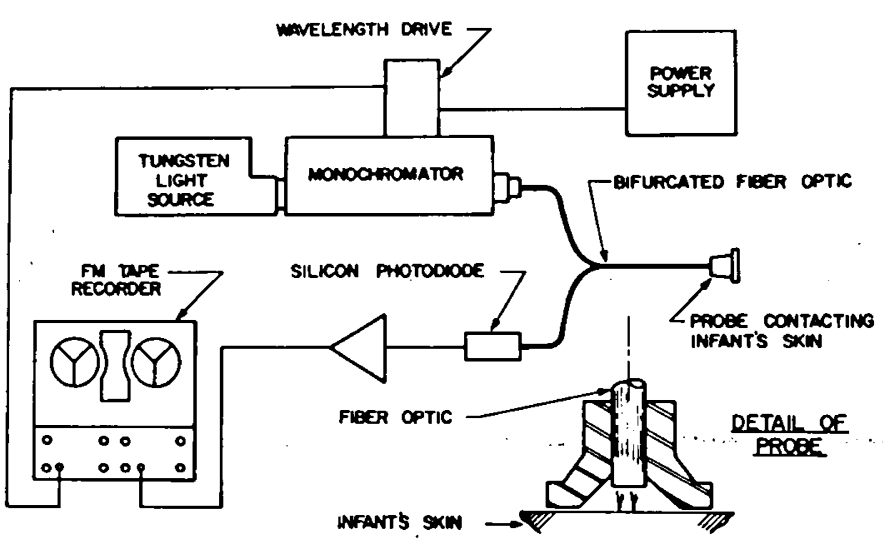

Fig. 1. Schematic of instrumentation for obtaining spectral reflectance of infant's skin.

After a complete verbal and written explanation of procedures, parental consent was obtained to perform spectral reflectance measurements on 56 Caucasian infants in the newborn nursery of a general hospital in Lafayette, IN. No non-Caucasian infants were available during the time of this study, but they are included in similar work currently being performed at a different institution.

Measurements of both blanched and unblanched skin were taken on the upper back. Serum bilirubin concentrations were determined by the Jendrassik microbilirubin method on samples drawn shortly after the reflectance measurements were made.

\section{RESULTS}

Of the 56 infants whose skin reflectance spectra were measured, a subset of 30 infants was found to be complete (no operator errors, missing bilirubin values, etc.) for analysis of skin reflectance spectra and corresponding serum bilirubin determination. Examples of these reflectance spectra and corresponding serum bilirubin values are presented in Figure 2. Note that near $460 \mathrm{~nm}$, the spectral reflectance of the skin decreases with increasing serum bilirubin concentrations. This accounts in part for the yellow appearance of the skin. These general observations are in agreement with previous studies $(1,2)$ of skin reflectance spectra change with level of jaundice. It was observed early in our study that the reflectance spectra of infants undergoing phototherapy exhibited marked differences from those not being so treated. Because of this, no infants who were currently or had been undergoing phototherapy were included in the analysis. This selection criteria is supported by Rubaltelli and Cavli (15) who observed cutaneous bilirubin concentrations to be different for these two groups. It was also observed that the individualities in epidermal hemoglobin concentration affected the skin reflectance spectra. Since blanching of the skin reduces the epidermal hemoglobin concentration, the statistical analysis was limited to only reflectance spectra of blanched skin.

The multispectral analysis of the data is based upon statistical regression analysis investigating the predictability of serum bilirubin level from reflectance spectra. First order linear, multiple linear, and polynomial nonlinear regressions were performed on the spectral reflectance values at each of the wavelength band intervals $(5 \mathrm{~nm})$ with respect to serum bilirubin level. The coefficients of determination $\left(R^{2}\right.$ values, a statistical measure of the closeness of fit of the data) for the regressions are summarized in Table 1 . The first order linear regression at the wavelength $450 \mathrm{~nm}$ has a $\mathrm{R}^{2}=0.783$ indicating approximately $78 \%$ of the variation in bilirubin level can be explained by a reflectance measurement at one single wavelength. The multiple linear regression analyses were performed on combinations of spectral reflectance values at specific wavelengths resulting in increased $R^{2}$ values. The best analysis results were obtained for a polynomial, nonlinear regression of the double logarithm of the reflectance spectra with respect to serum bilirubin concentration. For a combination of five wavelengths an $R^{2}$ value of 0.931 was obtained which implied that a relatively strong relationship existed between serum bilirubin concentration from skin reflectance. The ability to predict serum bilirubin concentration from skin reflectance using this relationship was tested using the original observations and is presented in Figure:3. The laboratory-determined serum bilirubin concentrations," denoted as "observed bilirubin value," are plotted on the ordinate with the concentrations predicted from the five wavelength double logarithm relationship on the abscissa. The $95 \%$ prediction limits for this empirical relationship was shown to be $\pm 2 \mathrm{mg} / 100 \mathrm{ml}$.

\section{DISCUSSION}

The development of the empirical model for the determination of serum bilirubin level from skin reflectance spectra was performed independently of any intuitive insight into which wavelengths contain information relevant to either serum or cutaneous bilirubin concentration. The statistical analysis confirmed anticipated results in that some of the significant wavelengths of the model were those which exhibit absorption characteristics peculiar to bilirubin and related compounds.

The work of Davis and Keohane (3) described a technique for measuring the change in optical properties of bilirubin in human sera during its initial degradation on exposure to light. The authors gave evidence of a decrease in absorption at $420 \mathrm{~nm}$ and $460 \mathrm{~nm}$ during this initial photo-oxidation period, implying that two bilirubin-related compounds exist in the serum. Others have observed that the absorption characteristics at $420 \mathrm{~nm}$ are attributable to free, unbound bilirubin in the serum whereas the characteristics at $460 \mathrm{~nm}$ are due to bilirubin bound to albumin (15). It is interesting to note that our study statistically predicted both $425 \mathrm{~nm}$ and $460 \mathrm{~nm}$ to be significant in the reflectance measurement of bilirubin.

There is no documentation verifying the presence of naturally occuring bilirubin related compounds that exhibit peculiar optical characteristics at 525,535 , or $545 \mathrm{~nm}$. It is therefore unlikely that the importance of these wavelengths arises from a bilirubin-related compound. A more plausible explanation is found in the absorption spectra of hemoglobin and the optical characteristics of the epidermis or serum proteins which have as yet not been investigated.

Hemoglobin exerts its effect on skin color by its cutaneous concentration. This is related to blood pressure, compression of the epidermis, and thermal characteristics of the skin. Although blanching tends to decrease the effect of hemoglobin, it does not eliminate it completely as is evidenced by the presence of

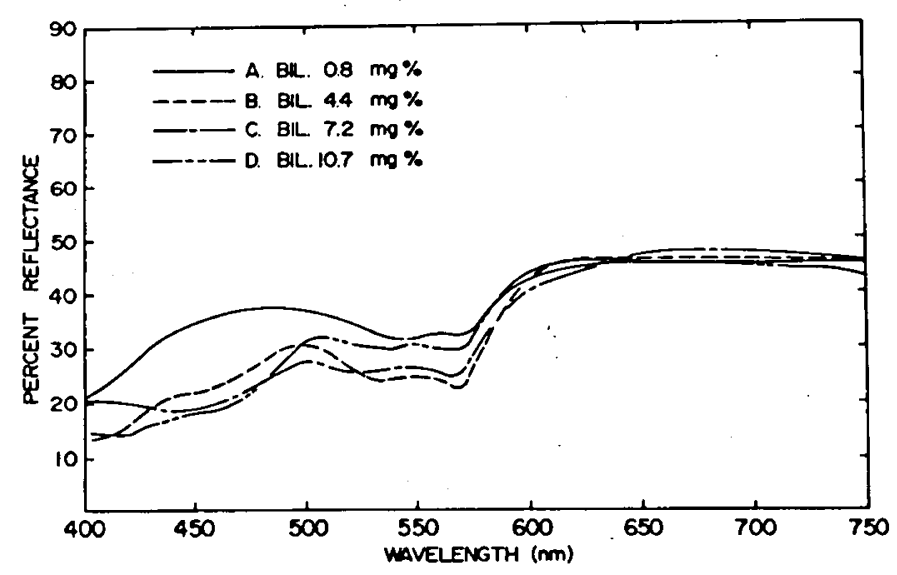

Fig. 2. Typical reflectance spectra and corresponding serum bilirubin concentration estimations. 
Table 1. Results of multispectral analysis of reflectance spectra and serum bilirubin

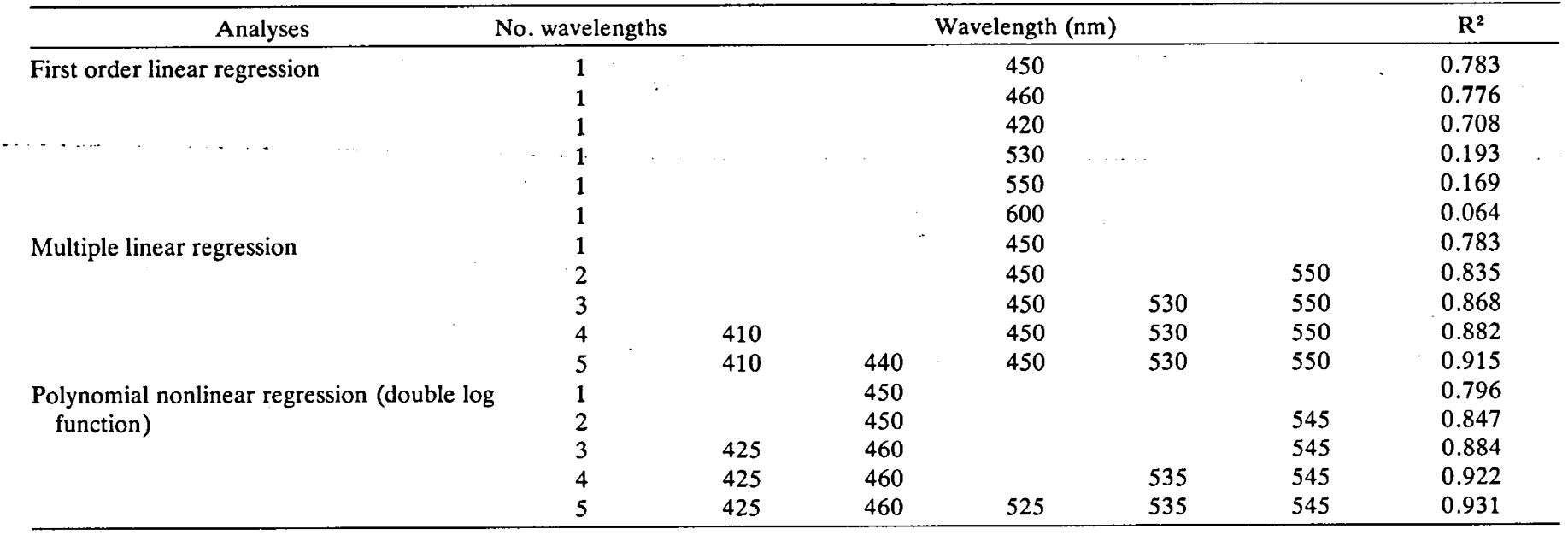

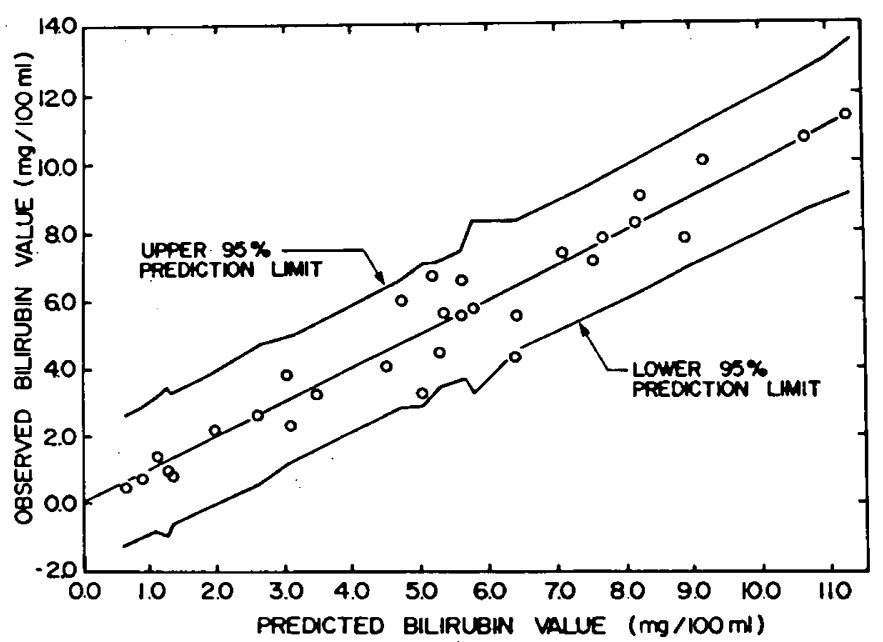

Fig. 3. The ability to predict serum bilirubin concentrations from skin reflectance using a five-wavelength model.

oxyhemoglobin absorption bands in the measured reflectance spectra, Figure 2 (2).

Total serum bilirubin level is not the best indicator of an infant's potential to develop kernicterus. Odell (12) and others $(11,13,16)$ have shown that the chemical determination of the reserve binding capacity of serum albumin for bilirubin might provide a better indication. Their explanation for this phenomena is simply that if bilirubin is bound to serum albumin, it will not diffuse into the brain and cause kernicterus. Safe levels of serum bilirubin are based upon the assumption that all infants have similar albumin concentrations and bilirubin kinetics, including intercellular transport, binding to albumin, and association with other serum proteins. This similarity between individuals does not always exist, however, and explains the development of kernicterus in infants whose total serum bilirubin levels had not even reached $10.0 \mathrm{mg} / 100 \mathrm{ml}(5)$.

Although other investigators have shown that the correlation between serum bilirubin level and cutaneous bilirubin concentration in infants not undergoing phototherapy is very small $\left(R^{2}=0.372\right)(1)$, this is felt to be acceptable for a screening technique and is certainly more accurate than visual estimations.

In addition to providing a screening method for neonatal jaundice, this device may also measure the potential for kernicterus. In a manner similar to the CNS, only unbound bilirubin in the blood will diffuse into the epidermis. Assuming that diffusion rates across CNS and epidermal cell membranes are governed by the concentration gradient of unbound bilirubin in the blood, cutaneous bilirubin concentration could correlate with bilirubin concentration in the CNS and thus reflect the potential for kernicterus.

\section{CONCLUSION}

A noninvasive method for serum bilirubin determination from neonatal skin reflectance has been demonstrated. Spectral reflectance measurements in the spectral region $400-750 \mathrm{~nm}$ were performed on the blanched (back) skin of 30 infants and a multispectral statistical regression analysis was made to investigate the predictability of serum bilirubin level. A relationship was found using the reflectance spectra at five wavelengths giving serum bilirubin prediction limits of $\pm 2 \mathrm{mg} / 100 \mathrm{ml}$ with a coefficient determination of 0.931 .

\section{REFERENCES AND NOTES}

1. Ballowitz, 1., and Avery, M. E.: Spectral reflectance of the skin. Biol. Neonate, 15: 348, (1970).

2. Brunsting, L. A., and Sheard,, C.: The color of the skin as analyzed by spectrophotometric methods II. The role of pigmentation. J. Clin Invest., 7: 575, (1929).

3. Davis, R. E., and Keohane, S. J.: Early changes in light-irradiated solutions of bilirubin; A spectrophotometric analysis. Photochem. Photobiol., 17: 302, (1973).

4. Edwards, E. A., and Duntley, S. Q.: The pigments and color of living human skin. Amer. J. Anat., 65: 1, (1939).

5. Gartner, L. M., Snyder, R. N., Chabon, R. S., and Bernstein, J.: Kernicterus: High incidence in premature infants with low serum bilirubin concentrations. Pediatrics, 45: 906 (1970).

6. Goldzieher, J. W., Roberts, I. S., Rawls, W. B., and Goldzieher, M. A.: "Chemical" analysis of the intact skin by reflectance spectrophotometry. AMA Arch. Derm. Syph., 64: 533 (1951).

7. Jacquez, J. A., and Kuppenheim, H. F.: Spectral reflectance of human skin in the region 235-1000 nm. J. Appl. Physiol., 7: 523 (1955).

8. Judd, D. B., and Wyszecki, G.: Color in Business, Science, and Industry, Ed. 2, pp. 106-158 (John Wiley \& Sons, Inc., New York, 1967).

9. Kuppenheim, H. F., and Heer, R. R., Jr.: Spectral reflectance of white and Negro skin between 440 and $1000 \mathrm{~nm}$. J. Appl. Physiol., 4: 800 (1952).

10. Lee, C. H.: The use of perspect icterometer for Korean neonates. J. Cath. Med. Coll., 20: 337 (1971).

11. Nakamura, H., and Lardinois, R.: Unbound bilirubin in icteric newborns. Biol. Neonate, 21: 400 (1972).

12. Odell, G. B.: Studies in kernicterus. I. The protein binding of bilirubin. J. Clin. Invest., 38: 823 (1959).

13. Odell, G. B., Stovey, G. N. B., and Rosenburn, L. A.: Studies in kernicterus III. The saturation of serum proteins with bilirubin during neonatal life and its relationship to brain damage at five years. J. Pediat., 76: 12 (1970).

14. Rowntree, L. G., and Brown, G. E.: A tintometer for the analysis of the color of the skin. Amer. J. Med. Sci., 170: 341 (1925).

15. Rubaltelli, F. F., and Cavli, M.: The effect of light on cutaneous bilirubin. Biol. Neonate, 18: 457 (1971)

16. Schiff, G. C. D., and Stern, L.: Competitive binding of free fatty acids and bilirubin to albumin: Differences in HBABA dye versus sephadex G-25 interpretation of results. Clin. Biochem., 4: 208 (1971).

17. Sheard C., and Brown, G. E.: The spectrophotometric analysis of the color of the skin. Arch. Intern. Med., 38: 816 (1926).

18. Thomas Ingram \& Co., Ltd.: A Perspect Icterometer for Neonates. Thomas Ingram \& Co., Ltd., Birmingham, England. 
19. Wasserman, H. P.: The color of human skin. Dermatologica, 143: 166 (1971).

20. Wiechel, J. F.: Optical detection of neonatal jaundice (Thesis, Master of Science, School of Mechanical Engineering, Purdue University, West Lafayette, Indiana, May 1975).

21. Dr. Hannemann is also Pediatrician, Arnett Clinic, Lafayette, IN.

22. Mr. Wiechel is presently affiliated with Mechanical Engineering Department;

0031-3998/78/1203-0207\$02.00/0

Copyright @ 1978 International Pediatric Research Foundation, Inc.
Ohio State University, Columbus, $\mathrm{OH}$.

23. This work was assisted by grants from the Biomedical Engineering Center, Purdue University, and from the NIAMDD, Grant RO1 AM18871.

24. Requests for reprints should be addressed to: Dr. R. E. Hannemann, School of Chemical Engineering, West Lafayette, IN 47907 (USA)

25. Received for publication February $28,1977$.

26. Accepted for publication June $28,1977$.

Printed in U.S.A. 